\title{
Functional Magnetic Resonance Imaging Shows Oxytocin Activates Brain Regions Associated with Mother-Pup Bonding during Suckling
}

\author{
Marcelo Febo, ${ }^{1}$ Michael Numan, ${ }^{2}$ and Craig F. Ferris ${ }^{1}$ \\ ${ }^{1}$ Department of Psychiatry and Center for Comparative NeuroImaging, University of Massachusetts Medical School, Worcester, Massachusetts 01655, and \\ ${ }^{2}$ Department of Psychology, Boston College, Chestnut Hill, Massachusetts 02467
}

\begin{abstract}
Oxytocin is released in the maternal brain during breastfeeding and may help strengthen the mother-infant relationship. Here, we used functional magnetic resonance imaging to determine whether oxytocin modulates brain activity in postpartum day $4-8$ dams receiving suckling stimulation. During imaging sessions, dams were exposed to pup suckling before and after administration of an oxytocin receptor antagonist. Another group of dams received oxytocin alone. Changes in brain activation in response to suckling closely matched that elicited by oxytocin administration. The overlapping brain areas included the olfactory system, nucleus accumbens, insular cortex, prefrontal cortex, ventral tegmental area, cortical amygdala, and several cortical and hypothalamic nuclei. Blockade of oxytocin receptors largely attenuated activation in these regions. The data suggest that oxytocin may strengthen mother-infant bond formation partly by acting through brain areas involved in regulating olfactory discrimination, emotions, and reward.
\end{abstract}

Key words: imaging; somatosensory; oxytocin; olfactory; maternal; lactation

\section{Introduction}

Breastfeeding is a unique form of social contact between a mother and her infant that promotes a strong affiliative bond. Studies in lactating rodents and sheep have advanced the understanding of the neurobiology of maternal behaviors (Numan, 1994; Kendrick et al., 1997). Focal lesions of the medial preoptic area, bed nucleus of the stria terminalis, lateral septum, amygdala, cingulate cortex, nucleus accumbens, and substantia nigra have demonstrated their role in the onset and/or maintenance of maternal behaviors (Slotnick and Nigrosh, 1975; Numan and Corodimas, 1985; Numan and Numan, 1996; Stack et al., 2002; Li and Fleming, 2003; Numan and Insel, 2003). Using cellular markers of gene expression, a more intricate network comprising a number of cortical, thalamic, hypothalamic, limbic, and brainstem sites has been identified (Kendrick et al., 1997; Lin et al., 1998; Lonstein et al., 1998; Li et al., 1999). Many of these brain areas express moderate to high levels of oxytocin receptors (Tribollet et al., 1988; Yoshimura et al., 1993; Veinante and Freund-Mercier, 1997; Vaccari et al., 1998), suggesting a convergence of maternal and oxytocinergic pathways. Elevated oxytocin receptor density has been correlated with greater levels of pup licking and grooming in postpartum dams (Francis et al., 2000; Champagne et al., 2001). Thus,

Received May 2, 2005; revised 0ct. 26, 2005; accepted 0ct. 27, 2005

This work was supported by from the National Institute on Drug Abuse Grant R01 DA13517 to C.F.F. and National Science Foundation Grant IBN 0312380 to M.N. We thank Tara Messenger for her help in conducting part of these experiments.

Correspondence should be addressed to Dr. Marcelo Febo, Department of Psychiatry and Center for Comparative Neurolmaging, University of Massachusetts Medical School, 55 Lake Avenue North, Worcester, MA 01655. E-mail: Marcelo.Febo@umassmed.edu.

DOI:10.1523/JNEUROSCI.3604-05.2005

Copyright $\odot 2005$ Society for Neuroscience $\quad 0270-6474 / 05 / 2511637-08 \$ 15.00 / 0$ the synaptic effects of oxytocin in the aforementioned structures may contribute to strengthening of mother-infant interactions.

Oxytocin synthesis mainly occurs in neurons of the paraventricular (PVN) and supraoptic nuclei (SON) of the hypothalamus. It is released from nerve terminals in the posterior pituitary and various regions of the brain. Suckling stimulates the release of oxytocin simultaneously into the bloodstream and CNS of postpartum rats (Neumann et al., 1993b). Systemically, this neurohypophyseal hormone enhances smooth muscle contractility, which is important for milk "let-down" during nursing. The release of oxytocin in the CNS during parturition initiates the onset of maternal behaviors. Expression of maternal behaviors in sheep and rats is delayed after blockade of oxytocin receptors during parturition (van Leengoed et al., 1987; Levy et al., 1992). Oxytocin release in response to suckling has been measured using microdialysis in the substantia nigra, olfactory bulbs, mediobasal hypothalamus, bed nucleus of the stria terminalis, medial preoptic area (MPOA), and septum of parturient sheep (Kendrick et al., 1997), as well as in sites of origin, the PVN and SON (Neumann et al., 1993a,b). Oxytocin release in the PVN and SON was blocked by an oxytocin receptor antagonist, therefore suggesting that this neuropeptide acts through a positive feedback mechanism.

The specific effect of oxytocin on brain activity after parturition, particularly during breastfeeding, remains unclear. Here, we tested whether oxytocin modulates suckling-stimulated brain activity in postpartum dams. Using blood oxygen level-dependent (BOLD) magnetic resonance imaging (MRI), we observed that continuous suckling from pups resulted in robust activation of brain areas involved in olfactory, emotional, and reward processing. Suckling-stimulated brain activation was paralleled by oxy- 
tocin (OT) administration and was partly blocked by an oxytocin receptor antagonist (OTA). Our present results provide evidence that oxytocin modulates brain activity during quiescent or motorically inactive suckling.

\section{Materials and Methods}

Subjects. Adult Sprague Dawley females were purchased from Charles River Laboratories (Charles River, MA). Rats were bred in the animal resource facilities of the University of Massachusetts Medical School. Only multiparous rats, having at least two litters, were included in the studies. Dams were housed with their litters in a temperature- and humidity-controlled room, under a $12 \mathrm{~h}$ light/dark cycle, with lights off at 6:00 P.M. Water and Purina (St. Louis, MO) rat chow were provided ad libitum. Animals were acquired and cared for in accordance with the guidelines published in the Guide for the Care and Use of Laboratory Animals (National Institutes of Health Publications No. 85-23, Revised 1985) and adhere to the National Institutes of Health and the American Association for Laboratory Animal Science guidelines. The protocols used in this study were in compliance with the regulations of the Institutional Animal Care and Use Committee at the University of Massachusetts Medical School.

Experimental groups and oxytocin administration. Experimental groups consisted of dams between postpartum days 4 (P4) and 8 (P8) that received suckling stimulation before $(n=7)$ and 45 min after blockade of oxytocin receptors $(n=7)$, and a second group of dams that received oxytocin $(n=6)$ or vehicle ( $10 \mu \mathrm{l}$ of artificial CSF; $n=4)$. A third group of dams $(n=4)$ were treated with oxytocin antagonist followed by suckling $45 \mathrm{~min}$ later to control for the possibility that the prolonged scanning session and experimental order of suckling first, followed by blockade and suckling, did not affect the imaging results. Injections of oxytocin or the receptor antagonist $\mathrm{d}\left(\mathrm{CH}_{2}\right)_{5}-\left[\mathrm{Tyr}(\mathrm{Me})^{2}, \mathrm{Thr}^{4}, \mathrm{Tyr}-\mathrm{NH}_{2}{ }^{9}\right]$ ornithine vasotocin (OTA) (Sigma, Natick, MA) were made via the intracerebroventricular (ICV) route while the animal was inside the magnet. Both chemicals were diluted in artificial CSF and injected at a dose of $1 \mu \mathrm{g}$ per $10 \mu \mathrm{l}$. The rationale and methods for ICV injections in rats during functional MRI has been described in detail previously (Febo et al., 2004). The ICV dose of oxytocin is twice the amount necessary to induce maternal behaviors in the virgin rat (Pedersen and Prange, 1979), and the dose of OTA has been shown to occupy most central oxytocin receptors for $6 \mathrm{~h}$ or more (Witt and Insel, 1991). Immediately before imaging, rats were anesthetized under $2.5 \%$ isoflurane, the skull surface was exposed, and the landmark suture bregma located. A 2.5\% lidocaine/2.5\% prilocaine cream was applied around the incision area. A 26-gauge cannula of polyethylene tubing (PE-10; inner diameter, $0.28 \mathrm{~mm}$; outer diameter, 0.61 $\mathrm{mm}$ ) was implanted into the lateral cerebral ventricle ( $1 \mathrm{~mm}$ caudal to bregma, $2 \mathrm{~mm}$ lateral to the midsagittal sinus, and $4 \mathrm{~mm}$ ventral to dura) and secured to the skull with surgical glue.

Suckling stimulation setup. Details of the setup for suckling stimulation are provided in Ferris et al. (2005) and illustrated in Figure 1. Briefly, four pups were placed in a weigh boat cradle padded with a double layer of gauze sheets (Fig. 1a). The pup cradle was positioned under a ventral opening in the body tube that allowed immediate access to two pairs of teats on each side of the dam's abdomen (for a total of 4 teats accessible to pups). Gliding a thin plastic sheet over the ventral opening of the body tube controlled access to teats (Fig. $1 b, c$ ). The end of the plastic sheet was easily accessible from outside of the bore of the magnet. The hindlimbs of dams were loosely tethered and raised just above the floor of the body tube. This provided a visual inspection of the ventrum from outside the magnet and prevented the dam from kicking and injuring pups during suckling. Pups used for the imaging session were separated from the dams for $\sim 45-60 \mathrm{~min}$ before the start of suckling stimulation. Our previous results indicated that pups attached to teats within seconds after a similar separation time (Ferris et al., 2005).

Functional imaging experiments. Studies were performed using a multiconcentric dual-coil small animal restrainer (Insight NeuroImaging Systems, Worcester, MA), and described in detail in Ludwig et al. (2004). Before breeding, females were acclimated to the restrainer and the imaging protocol as described previously by Febo et al. (2004). Briefly, animals
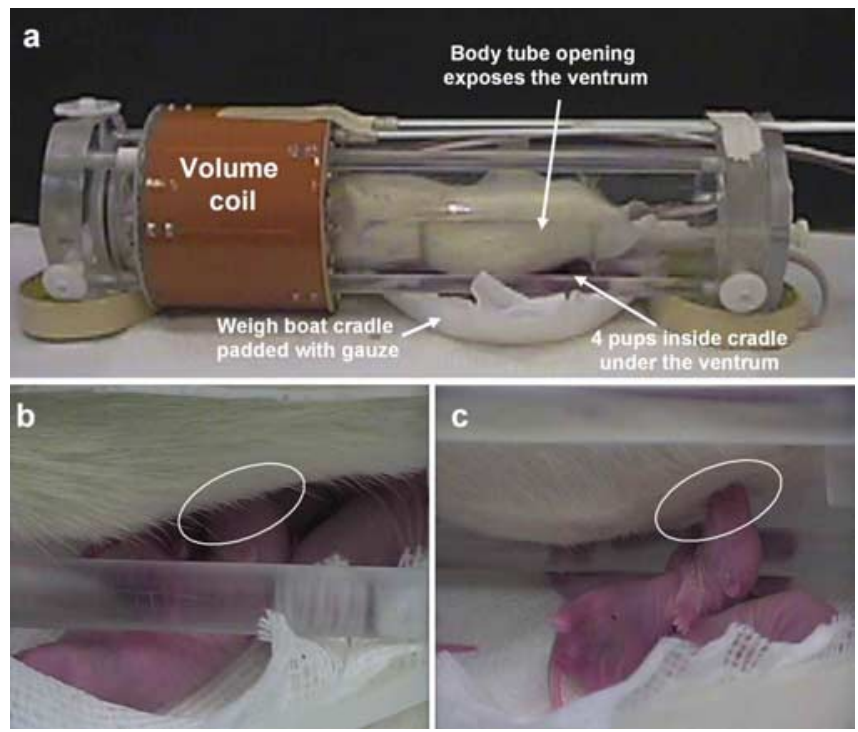

Figure 1. Experimental setup for imaging awake postpartum dams during suckling. $\boldsymbol{a}, \mathrm{A}$ body tube opening allows pups to gain access to two pairs of teats on either side of the ventrum. Pups are placed under the chassis at a close distance. $\boldsymbol{b}$, Contact with the ventrum and teat is established within seconds during a functional scan. c, Side view showing a suckling pup.

were lightly anesthetized with $2 \%$ isoflurane and secured into the dualcoil restrainer. When fully conscious, the restraining unit was placed into a black opaque tube that served as a "mock scanner" and a tape recording of an MRI pulse sequence played for $90 \mathrm{~min}$ to simulate the bore of the magnet and an imaging protocol. This procedure was repeated over three consecutive days. Results obtained from our laboratory indicate that this procedure improves contrast-to-noise and signal-to-noise ratios because of reduced motor episodes during imaging sessions (King et al., 2005).

Functional imaging was conducted in a Bruker Biospec $4.7-\mathrm{T} / 40 \mathrm{~cm}$ horizontal magnet (Oxford Instruments, Oxford, U.K.) equipped with a Biospec Bruker console (Bruker, Billerica, MA) and a $20 \mathrm{G} / \mathrm{cm}$ magnetic field gradient insert (inner diameter, $12 \mathrm{~cm} ; 120 \mu$ s rise time). To reduce discomfort from ear and nose bars, a topical anesthetic (2.5\% lidocaine/ $2.5 \%$ prilocaine cream) was applied to skin and soft tissue in the ear canal and over the bridge of the nose. Injections were made via a plastic syringe connected at the end of the ICV cannula tubing. Cannula placement was verified before cocaine imaging with a short anatomical MR scan. Only animals with correct placement were included in the study.

Functional images were all obtained with a BOLD-weighted multislice fast spin echo pulse sequence with the following parameters: 14 slices, 1.2 $\mathrm{mm}$ thick; field of view, $30 \mathrm{~mm}$; $64 \times 64$ data matrix; echo train length (ETL), 16; echo spacing, $7 \mathrm{~ms}$; repetition time (TR), $2108 \mathrm{~ms}$; effective echo time $\left(\mathrm{TE}_{\mathrm{eff}}\right), 53.2 \mathrm{~ms}$. A single acquisition of all 14 slices took $8.4 \mathrm{~s}$. For suckling stimulation, 110 repetitions were acquired for a total scan time of $15 \mathrm{~min}$. After a $5 \mathrm{~min}$ baseline, pups were allowed access to teats for the remaining $10 \mathrm{~min}$. Suckling was terminated by replacement of the plastic partition. A high-resolution anatomical scan was collected for 13 min using a fast spin echo pulse sequence $\left(\mathrm{TE}_{\mathrm{eff}}, 48 \mathrm{~ms}\right.$; TR, $2500 \mathrm{~ms}$; field of view, $30 \mathrm{~mm}$; $1.2 \mathrm{~mm}$ slice thickness; $256 \times 256$ data matrix; ETL, 16; 12 averages). After anatomy acquisition, dams were given an ICV injection of OTA. After a $40 \mathrm{~min}$ wait, the second suckling bout was imaged in the presence of oxytocin receptor blockade. A separate group of P4-P8 dams $(n=4)$ were given ICV OTA 45 min before suckling stimulation to control for the prolonged imaging session and experimental order of suckling followed by oxytocin blockade/suckling. For oxytocin administration, $\mathrm{P} 4-\mathrm{P} 8$ dams were given an ICV injection of oxytocin during a 30 min functional scan (oxytocin injected at $5 \mathrm{~min}$ ). After this scan, a highresolution anatomical scan was collected.

A separate group of nonmaternal male rats were used to test the effects of OTA administration on general arousal. Recently, oxytocin has been reported to modulate sleep/wake states in rats (Lancel et al., 2003). Thus, the additional control group $(n=4)$ was included to examine whether 
a

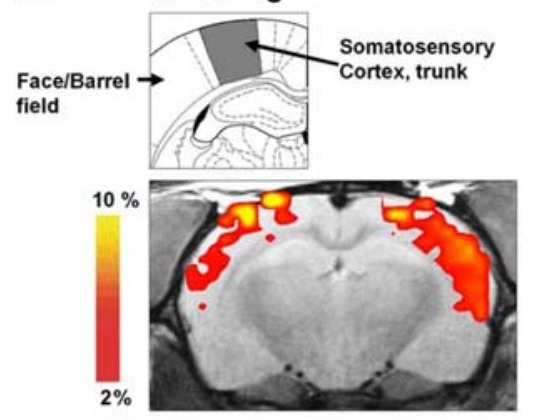

C

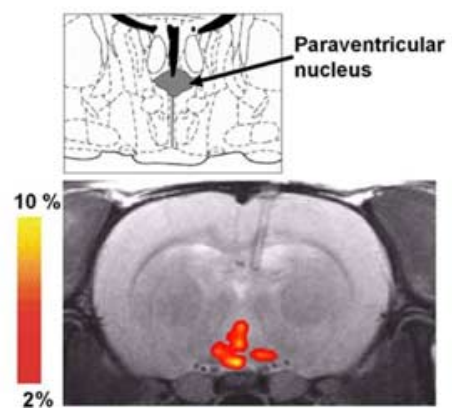

b

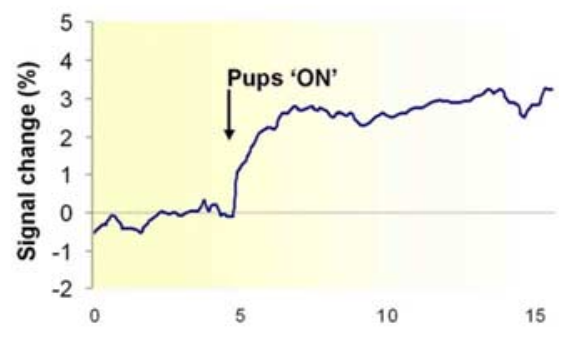

Minutes

d

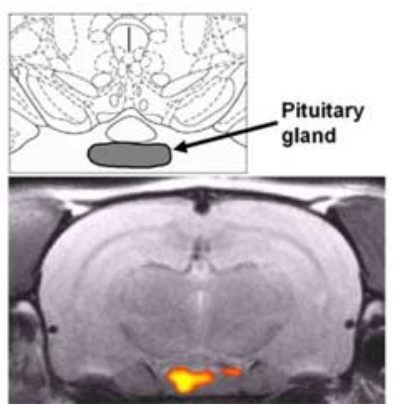

Figure 2. Brain regions used for validating the suckling-stimulation paradigm during functional imaging. Atlas coronal sections and representative functional images highlight the somatosensory cortex $(\boldsymbol{a})$, paraventricular nucleus $(\boldsymbol{c})$, and pituitary (d) activated with suckling. Scale bar hue indicates the percentage-change value. $\boldsymbol{b}$, BOLD signal change over time during suckling stimulation (expressed as percentage change from baseline).

algorithm with automated linearization to create accurate 3D surface shells for each subject anatomy (Wu and Sullivan, 2003). These anatomy shells are aligned to the atlas shell. The affined registration involved translation, rotation, and scaling in all three dimensions, independently.

Statistical $t$ tests were performed on each subject within their original coordinate system. The control window was the first 35 scan repetitions for all groups, except for the hindpawstimulation experiments, which consisted of three control epochs of five repetitions each. The stimulation window was 65 repetitions for suckling stimulation, 150 repetitions for oxytocin or vehicle administration and three stimulation epochs of five repetitions for the hindpaw stimulation. Statistical $t$ tests used a $95 \%$ confidence level, two-tailed distribution, and heteroscedastic variance assumptions. To provide a conservative estimate of significance, a falsepositive detection-controlling algorithm was introduced into the analysis (Genovese et al., 2002). This ensured that the false-positive detection rate was $<5 \%$. Statistically significant pixels were assigned their percentage change values (stimulus mean minus control mean) and all other pixels were set to zero. Statistical composite maps were created for each group. A Bonferroni correction factor was used to maintain an overall uncertainty level of $p=0.05$. Multiple comparisons among the three groups were performed using a nonparametric Kruskal-Wallis test.

\section{Results}

the effects of OTA on a sensory-arousing stimulus was partly caused by alterations in wakefulness of rats. Rats were set up for imaging as indicated above (see above, Functional imaging experiments). One copper wire electrode was placed on each hindpaw and adhered in place with medical gauze tape. Electrodes extended outside the magnet bore and were connected to an isolated pulse stimulator (A-M systems, Carlsborg, WA) that controlled the delivery of $1 \mathrm{~mA}$ electrical pulses to animals during scanning. Functional images were collected using the same parameters mentioned above; however, three sequential baselinestimulation block design epochs of $40 \mathrm{~s}$ each were collected. For experiments, rats were first tested for their neural response to hindpaw stimulation, and then they received an ICV injection of OTA ( $1 \mu$ g per 10 $\mu \mathrm{l})$. Forty-five minutes later, animals were again tested for their responsiveness to peripheral hindpaw stimulation.

Data analysis. Data analysis involved several steps: evaluation of individual datasets for artifacts, coregistration of datasets to a multisegmented three-dimensional (3D) rat brain atlas, and statistical analysis to evaluate stimulus-evoked signal changes per each region of interest per subject. Anatomical and functional images are evaluated for artifacts using Stimulate (Strupp, 1996). Motion artifact in the functional scans was assessed by (1) subtraction of the anatomical data across the imaging session, (2) qualitative analysis of time-series movies looking for voxel displacement, and (3) analysis of the raw data time series for course spikes. The time-series movies correlated with course spike activity. Individual course spikes displaced above or below 1 SD of the time series baseline average were excluded.

Details of the alignment of scans to the rat brain atlas have been published previously (Wu and Sullivan, 2003; Ferris et al., 2005). Each individual scan is aligned to a fully segmented rat brain atlas that delineates $>1200$ distinct anatomical subvolumes with the brain based on twodimensional atlas textbooks (Paxinos and Watson, 1997; Swanson, 1999). These detailed regions are collected into 12 major regions of the brain. (e.g., cortex, thalamus, amygdaloid complex, cerebrum, cerebellum, hypothalamus, etc.). The alignment process used a marching cube

\section{Suckling-induced brain activation in lactating rats}

Regions of interest showing positive BOLD activation represent brain voxels in which signal intensity during pup suckling was significantly greater than the baseline signal ( $t$ test analysis; $p<$ $0.05)$. Activation of the somatosensory cortex (SSCtx), PVN, and pituitary were considered reliable indicators of pup-suckling stimulation (Fig. 2). As shown in Figure $2 a$, suckling strongly activated the SSCtx, specifically in areas corresponding to the trunk and barrel field regions. Suckling evoked an immediate rise in BOLD signal intensity that peaked at a $3 \%$ signal increase (SD $\pm 0.3 \%$ ) within seconds (Fig. 2b). Similarly, the pituitary and PVN showed increases in BOLD signal intensity that followed the same temporal activation pattern (Figs. $2 c, d$ ). Robust activation was observed in the olfactory bulbs $(\mathrm{OB})$, and heavy clustering of positive BOLD voxels was observed in brain areas that receive direct synaptic inputs from the OB. These included the piriform cortex (PC), olfactory tubercle (Olf Tub), anterior olfactory nucleus (AON), insular cortex (IC) and cortical amygdala (Fig. 3). Suckling activated other cortical and subcortical structures: the dentate gyrus (DG), ventral subiculum (SUB), hippocampal CA1 and CA3 regions, entorhinal cortex (EC), septum, ventromedial nucleus, PVN, MPOA, lateral hypothalamus (LH), nucleus accumbens (NA), ventral pallidum (VP), the medial and lateral prefrontal cortices (PFC), the auditory cortex (Aud Ctx), the caudate-putamen $(\mathrm{CPu})$, substantia nigra $(\mathrm{SN})$, the ventral tegmental area (VTA), and the periaqueductal gray (PAG). It is important to mention that regions of the parietal and temporal cortices showed positive BOLD activation during suckling (Fig. 3). Negative BOLD-signal changes were scantily distributed in several cortical regions. No significant signal decreases were observed in subcortical areas. 
Effect of oxytocin receptor blockade on suckling-evoked brain activity

Blocking oxytocin receptors with OTA resulted in attenuated positive BOLD responses to suckling, in comparison with the response before the antagonist (Fig. 3). Considering that this effect could potentially result from loss of general alertness during the $1.5 \mathrm{~h}$ imaging sessions, a group of dams were tested 45 min after administration of OTA $(n=4)$. Using this design to counterbalance the effects of prolonged imaging, we observed that the PVN, MPOA, and pituitary showed a dramatic reduction in positive BOLD activation when compared with suckling without OTA $(p<0.03$, using a Kruskal-Wallis test). Interestingly, oxytocin receptor blockade showed a strong effect for regions of the olfactory system $(p<0.01$ vs suckling before OTA; Kruskal-Wallis test). The specific brain regions consistently showing a reduced positive BOLD response to suckling with OTA were as follows: the IC $(p=0.04), \mathrm{PC}(p=0.04), \mathrm{AON}(p=0.03)$, Olf Tub $(p=0.04)$, the DG $(p=0.05)$, CA1 $(p=0.05), \mathrm{CA3}(p=0.04)$, Aud Ctx $(p=$ $0.01), \mathrm{SN}(p=0.05)$, and the lateral PFC $(p=0.05)$. Data for several of these regions are shown in Figure 4 or summarized in Table 1. Among the regions of interest that did not show a reduced positive BOLD response to suckling with OTA were as follows: the $\mathrm{NA}, \mathrm{CPu}, \mathrm{VP}, \mathrm{LH}$, septum, amygdala, and PAG. OTA administration was not observed to increase the BOLD response to suckling.

Effect of oxytocin receptor blockade on evoked cortical activity

The overall effect of OTA administration before suckling was a reduction in the number of activated voxels in brain areas in the section above. It is reasonable to believe that this effect might arise from nonspecific changes in sensitivity to sensory arousing stimuli and not only to the suckling stimulus. Thus, the effectiveness of OTA in modulating overall, general wakefulness in rats was evaluated by determining the number of cortical voxels activated by an arousing stimulus before and after OTA administration. Data for the cerebral cortex are summarized in Figure 5. Blockade of oxytocin receptors did not result in changes in the number of cortical voxels activated by hindpaw electrical stimulation (before OTA, $118 \pm 6$; after OTA, $125 \pm 1.5)$.

\section{Oxytocin-induced brain activation in lactating rats}

The effects of ICV oxytocin administration were tested against the effects of vehicle injection. Vehicle injections resulted in positive BOLD activity, unilaterally in one of the lateral cerebroven-

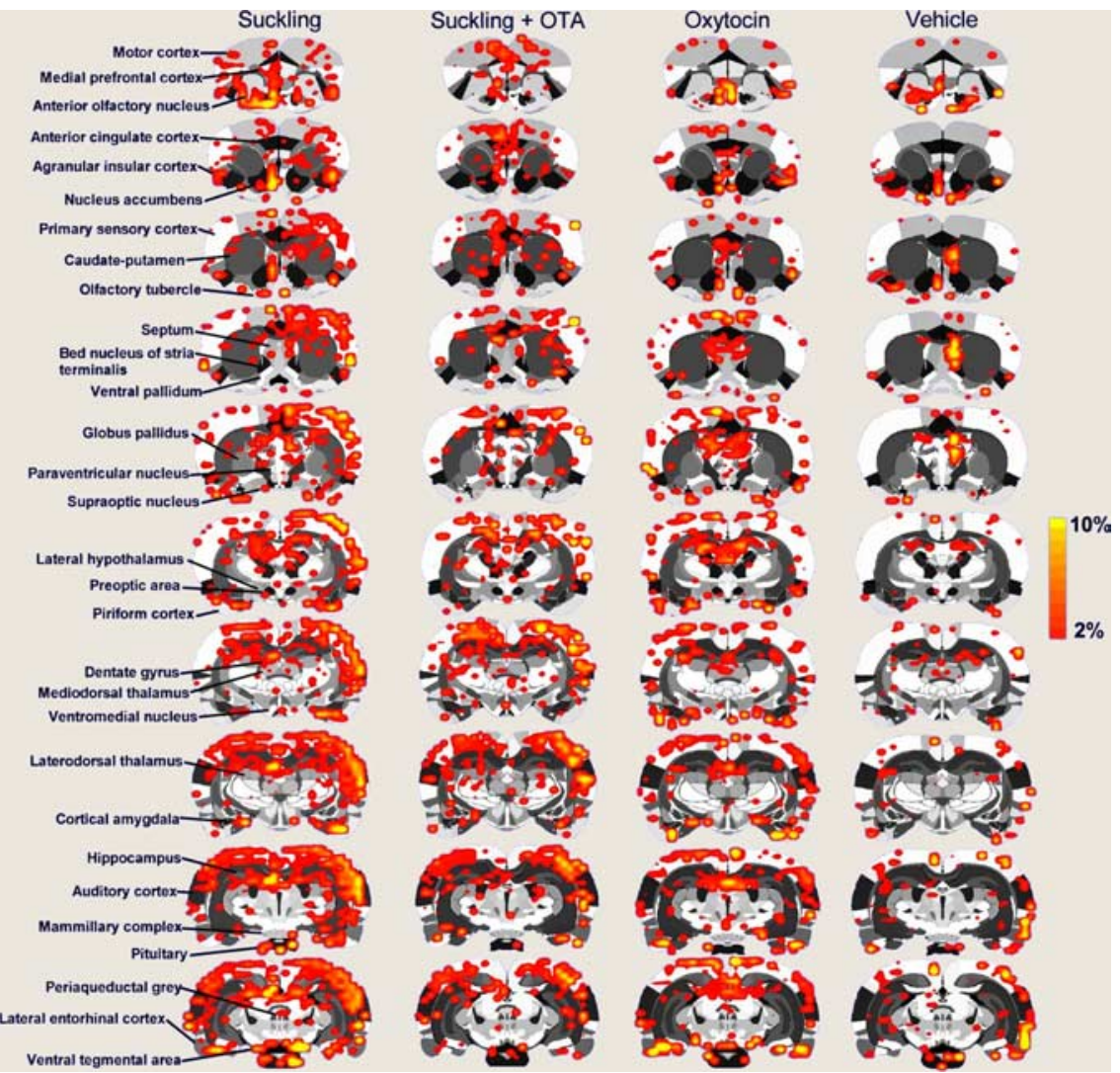

Figure 3. Two-dimensional statistical maps of brain activation in response to suckling or oxytocin. Activation maps are composites of P4-P8 dams that received suckling stimulation before and after oxytocin receptor blockade or oxytocin and vehicle administration. Colored pixels represent brain areas that showed signal intensity values significantly increased above baseline. Scale bar hue indicates the percentage-change value. Statistical significance was determined using a voxel-wise $t$ test analysis with false-positive detection filtering, comparing baseline and stimulation periods ( $\alpha=0.05$; Bonferroni corrected). Various regions of interest are indicated to the left.

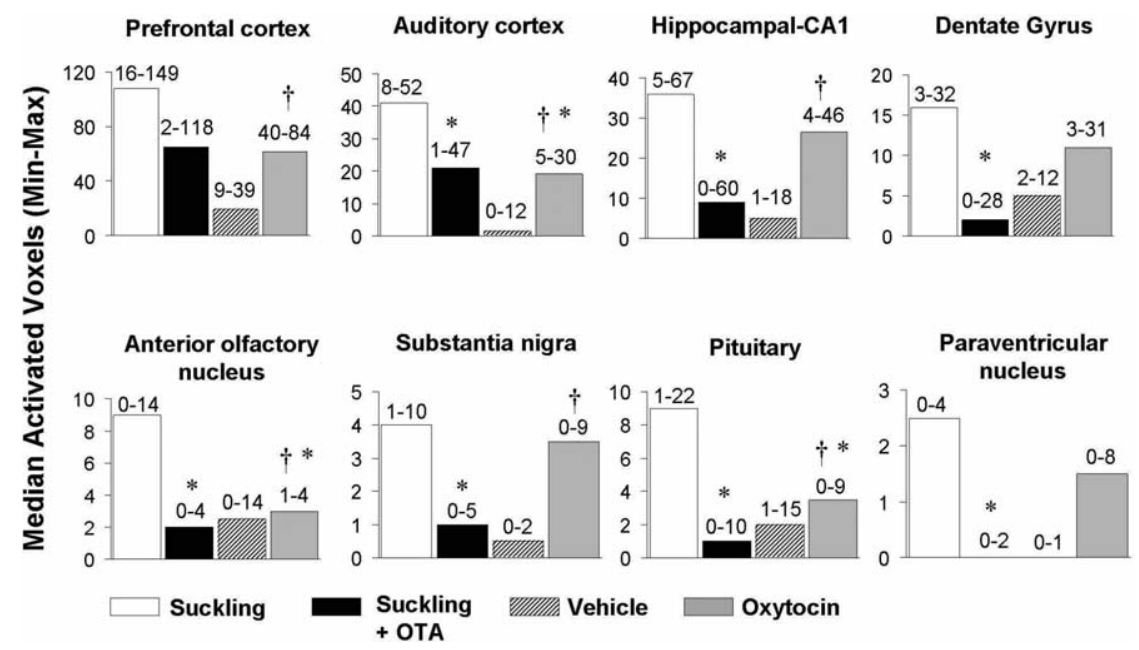

Figure 4. Median number of voxels activated by suckling, oxytocin, or vehicle administration. Data are shown for P4 -P8 dams that received suckling stimulation before (empty bars) and after (dark bars) oxytocin receptor blockade, or with oxytocin (gray bars) and vehicle (patterned bars) alone. Numbers on top of the bars indicate the data range across individual animals (minimum and maximum). Symbols indicate statistical differences at $p<0.05$ using a nonparametric Kruskal-Wallis test. * Significantly different from suckling alone. ${ }^{\dagger}$ Significantly different from vehicle.

tricles, near the rostral rhinal incisura, and caudally near the rhinal fissure (Fig. 3). Oxytocin produced a specific pattern of brain activation not presented by the vehicle (Fig. 3). Oxytocininduced brain activation shared similarities with the anatomical 
Table 1. Positive BOLD signal changes following suckling, oxytocin, or vehicle

\begin{tabular}{|c|c|c|c|c|}
\hline Region of interest & Suckling before 0TA & Suckling after OTA & 0xytocin & Vehicle \\
\hline \multicolumn{5}{|l|}{ Cortical areas } \\
\hline Insular & $23(3-47)$ & $13(0-20)^{*}$ & $13(6-27)$ & $6(2-30)$ \\
\hline Cingulate & $15(0-21)$ & $6(0-25)$ & $11(0-22)^{* *}$ & $3(2-4)$ \\
\hline Parietal & $20(11-33)$ & $17(0-40)$ & $9(0-28)^{* * * * *}$ & $1(0-6)$ \\
\hline Somatosensory & $150(47-238)$ & $73(13-227)$ & $80(55-177)^{* * *}$ & $26(11-48)$ \\
\hline Temporal & $23(10-40)$ & $13(2-42)$ & $17(7-21)^{* *}$ & $7(4-11)$ \\
\hline Piriform & $29(6-71)$ & $12(1-25)^{*}$ & $18(6-38)$ & $9(5-75)$ \\
\hline Prelimbic & $14(0-34)$ & $12(0-22)$ & $7(1-13)$ & $3(0-18)$ \\
\hline \multicolumn{5}{|l|}{ Hypothalamic areas } \\
\hline Preoptic area & $4(0-7)$ & $0(0-2)^{*}$ & $3(0-10)^{* *}$ & $0(0-0)$ \\
\hline Lateral hypothalamus & $11(2-29)$ & $5(0-14)$ & $6(1-12)$ & $5(0-9)$ \\
\hline Anterior hypothalamus & $1(0-2)$ & $0(0-2)$ & $1(0-2)$ & $0(0-0)$ \\
\hline Posterior hypothalamus & $0(0-4)$ & $0(0-2)$ & $1(0-3)$ & $0(0-0)$ \\
\hline \multicolumn{5}{|l|}{ Amygdala areas } \\
\hline Cortical & $9(4-21)$ & $12(0-29)$ & $13(5-14)$ & $5(3-13)$ \\
\hline Central & $1(0-5)$ & $1(0-3)$ & $2(0-2)$ & $0(0-1)$ \\
\hline Posterior & $0(0-3)$ & $0(0-2)$ & $1(0-4)$ & $0(0-1)$ \\
\hline Caudate-putamen & $58(9-113)$ & $26(0-70)$ & $15(5-49)^{*, * *}$ & $1(0-12)$ \\
\hline Nucleus accumbens & $5(0-14)$ & $3(0-13)$ & $2(0-6)$ & $1(0-2)$ \\
\hline Ventral pallidum & $5(1-6)$ & $2(0-6)$ & $2(0-8)$ & $0(0-3)$ \\
\hline Ventral tegmental area & $3(0-7)$ & $2(0-6)$ & $2(0-4)^{* *}$ & $0(0-2)$ \\
\hline Olfactory bulbs & $28(0-47)$ & $11(0-30)$ & $7(0-15)^{*}$ & $8(3-10)$ \\
\hline Olfactory tubercle & $8(1-10)$ & $1(0-14)^{*}$ & $6(3-11)$ & $4(0-29)$ \\
\hline Periaqueductal gray & $2(0-8)$ & $1(0-14)$ & $3(0-12)$ & $1(1-10)$ \\
\hline Septum & $10(1-13)$ & $6(0-12)$ & $7(1-19)$ & $8(3-15)$ \\
\hline \multicolumn{5}{|l|}{ Hippocampal areas } \\
\hline CA2 & $1(0-1)$ & $1(0-3)$ & $0(0-1)$ & $0(0-1)$ \\
\hline CA3 & $17(5-34)$ & $6(0-34)^{*}$ & $7.5(4-19)^{*}$ & $8(4-19)$ \\
\hline Subiculum & $22(4-39)$ & $4(0-55)$ & $13(3-35)$ & $6(1-22)$ \\
\hline
\end{tabular}

Data are expressed as median number of voxels activated relative to baseline (range in parentheses). Results are for P4-P8 dams that received suckling stimulation before $(n=7)$ and after oxytocin receptor blockade $(n=9)$, or with oxytocin $(n=6)$ and vehicle $(n=4)$.

*Significantly different from suckling. **Significantly different from vehicle. $p<0.05$ using a nonparametric Kruskal-Wallis test. for the following regions: PVN, olfactory tubercle, anterior olfactory nucleus, insular cortex, piriform cortex, cortical amygdala, medial preoptic area and prefrontal cortex. For each of these regions, suckling and ICV oxytocin administration increased the BOLD signal. In addition, OTA administration blocked the sucklinginduced increase in the BOLD signal. Several of these areas have been associated with the olfactory system, either indirectly or by direct projections received from the olfactory bulbs (Price, 1973). This suggests that OT release during nursing contributes to olfactory-related neural activity. It is important to mention, however, that changes in BOLD signal intensity do not measure directly the release of OT, but the similarities in brain activation patterns observed with suckling and OT suggest that a component of suckling-stimulated activity is associated with OT. This is further supported by blockade with OTA. BOLD signal changes do correspond, albeit indirectly, to alterations of cerebral blood flow associated with increased neuronal activity. Thus, one conclusion could be that suckling and oxytocin directly affect local cerebral hemodynamics vis-à-vis neuronal activity. Whether these changes relate to inhibitory or excitatory neurotransmission remains unknown.

The results for the PVN are not surprising because the PVN is involved in the pattern of activity evoked by suckling (Fig. 3) and was consistent with the distribution of oxytocin receptors reported in the literature (Fig. 6) (Tribollet et al., 1988; Yoshimura et al., 1993; Veinante and Freund-Mercier, 1997; Vaccari et al., 1998). The temporal dynamics of oxytocin-induced brain activation showed a delayed onset, reaching a plateau positive BOLD response of $3 \%$ $(\mathrm{SD} \pm 0.5 \%)$ at $\sim 10 \mathrm{~min}$ postinjection, which remained toward the end of the scan. Brain areas activated by oxytocin in comparison to vehicle were as follows: the temporal cortex, CA1, Aud Ctx, SN, SSCtx, PFC, CPu, DG, cingulate cortex, parietal cortex, POA, and PVN. No statistical differences in these brain structures were found between suckling stimulation and oxytocin, with the exception of the $\mathrm{CPu}$, the parietal and auditory cortices, $\mathrm{AON}$, OBs and pituitary, which did show a lower positive BOLD response to oxytocin than suckling $(p<0.05)$ (Figs. 3, 4).

To summarize, suckling and oxytocin administration coincided in activating the AON, dorsolateral and medial PFCs, NA, VTA, IC, PC, Olf Tub, cingulate cortex, parietal cortex, MPOA, VMH, PVN, cortical amygdala, EC, DG, PAG and SUB. The CPu, parietal and auditory cortices, $\mathrm{AON}, \mathrm{OBs}$, and pituitary showed a greater BOLD response with suckling than oxytocin. OTA reduced positive BOLD responses to suckling stimulation in the pituitary, MPOA, PVN, IC, AON, Olf Tub, DG, CA1, CA3, Aud Ctx, SN, and the lateral PFC, but not the LH, septum, or PAG (Table 1).

\section{Discussion}

In this paper we present evidence that suckling-induced OT release into central neural sites increases the BOLD signal in many areas of the postpartum rat brain. The best evidence is provided milk-ejection reflex, and OT exerts a positive feedback effect on its own release from PVN neurons (Neumann et al., 1994). Importantly, the activated PVN is likely to be releasing OT into central neural sites, as well as into the periphery via the posterior pituitary. In the rat, destruction of the PVN delays the onset of maternal behaviors (Insel and Harbaugh, 1989). Similar effects have been observed by administering OTA (van Leengoed et al., 1987). Our present data provide evidence that oxytocin continues to be released within this area in fully maternal postpartum rats. With respect to the olfactory-related areas (Olf Tub, AON, insular, etc.), the increased BOLD signal in these areas may be related to aspects of maternal-behavior control. Naive virgin female rats are not maternally responsive but instead avoid pups and find their odors aversive (Fleming et al., 1979), whereas postpartum rats are attracted to pups and their odors (Numan and Insel, 2003). Perhaps the increased BOLD signal observed in these regions as a result of suckling-induced OT release into the brain maintains the postpartum functional change in the olfactory system so that pup-odors continue to be attractive.

In seeming contradiction to this point of view, it is known that once maternal behavior has become established, acute ICV injection of OTA does not cause a major disruption of maternal behavior in postpartum rats. However, it is possible that a longterm disruption of central oxytocinergic effects would be more disruptive (Pedersen et al., 1995; Pedersen and Boccia, 2003). It should also be mentioned that the central release of OT might be related to maternal aggression. The heightened aggressiveness shown by postpartum females is dependent on ventral/suckling stimulation from pups (Numan, 2004). Alterations in olfactory 
processing might play a role in this increased maternal aggressiveness aimed at intruders at the nest site (Numan and Insel, 2003).

The increased BOLD signal in the MPOA as a result of suckling-induced OT release into the brain may also be related to maternal behavior. An intact MPOA is essential for maternal behavior, and estradiol and prolactin act on the MPOA to stimulate maternal behavior (Numan and Insel, 2003). In addition, direct OTA administration into the MPOA blocks the onset of maternal behavior in parturient rats (Pedersen et al., 1994). However, it is also possible that MPOA projections to the PVN promote OT release from the pituitary as part of the milk-ejection reflex ( $\mathrm{Si}$ merly and Swanson, 1988; Numan and Numan, 1996). The reason we raise this latter possibility is because of the fact that MPOA lesions tend to disrupt the motorically active components of maternal behavior (retrieving and nest building), while leaving nursing behavior relatively unaffected (Numan and Insel, 2003). It is certainly possible, however, that OT release into the MPOA during nursing in some way maintains the functional integrity of the MPOA so that it can control active maternal responses once a nursing bout terminates.

An important part of the present results is the activation of a reward system by suckling and oxytocin. This is consistent with our previous work demonstrating a robust activation of the VTA-accumbensprefrontal cortex pathway with suckling (Ferris et al., 2005). Several lines of evidence show that pups are reinforcing for early postpartum rats. Before postpartum day 10 , females will prefer to spend more time in a pup versus cocaine-paired environment, suggesting that the rewarding properties of pups during early postpartum can outweigh those of cocaine (Mattson et al., 2001). Dams have also been shown to lever press for pups, a behavior reduced by selective lesions of the MPOA and Amyg (Lee et al., 1999). Activity within the VTA-accumbens-prefrontal cortex pathway can thus be expected to play a part in accentuating the rewarding nature of pups, thereby reinforcing maternal behaviors. Neuronal activity in the accumbens, at least during parturition, plays a role in developing and retaining the active or motoric components of maternal behaviors (Lee et al., 1999), and dopamine in this brain area has been linked to retaining maternal responsiveness (Byrnes et al., 2002). We observed activation of the accumbens of dams with suckling, which is probably associated with the release of dopamine during interactions with pups (Hansen et al., 1993). The fact that we also observed accumbens activation with OT could suggest that this neuropeptide somehow induces the release of dopamine within this region, and perhaps the prefrontal cortex. Results showing evidence of oxytocin receptor density in the accumbens has not been consistent (Tribollet et al., 1988; Veinante and Freund-Mercier, 1997), whereas its messenger
After OTA
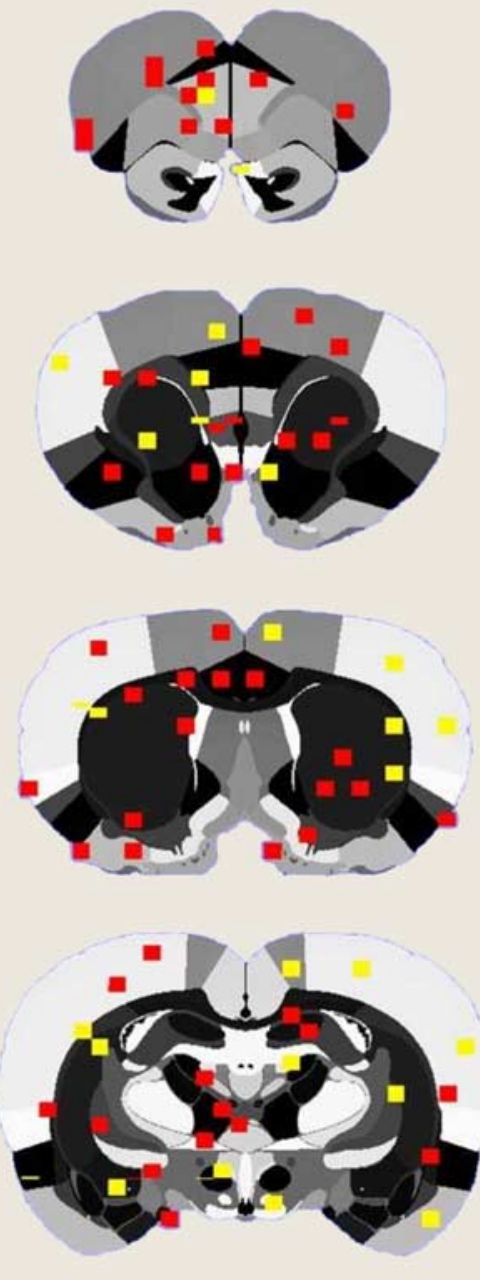

Figure 5. Effect of oxytocin or an oxytocin antagonist on evoked cortical activity. Compositive BOLD activation maps in rats given electrical $(1 \mathrm{~mA})$ stimulation of the hindpaw both before and after blockade of oxytocin receptors with an antagonist are shown. The colored pixels represent statistically significant increases in BOLD signal ( $p<0.05 ; t$ test).

RNA has not been clearly observed in this area (Yoshimura et al., 1993; Vaccari et al., 1998). Thus, it is possible that suckling and the effect of OT on neuronal activity in the accumbens, as suggested by the present data, may be mediated indirectly via the VTA and/or MPOA (Numan and Smith, 1984; Pedersen et al., 1994).

In a recent study (Numan et al., 2005), it was proposed that MPOA projections to the VTA are important for the active components of maternal behavior. It was also proposed that activation of the VTA by the MPOA causes the VTA to release dopamine into the NA and to inhibit the output of NA. In other words, an increase in MPOA and VTA output and a decrease in NA output were viewed as being important for active, voluntary maternal responses. Nursing activity, which is a motorically inactive state induced by suckling stimulation (Lonstein and Stern, 1997), might be expected to be associated with decreased output of the VTA and increased output of the NA. The increased BOLD signal in the NA after suckling stimulation or OT administration (interestingly, this response was not blocked by OTA) would conform to this motor inhibition hypothesis. With respect to the VTA, the increased BOLD response induced by suckling (which 


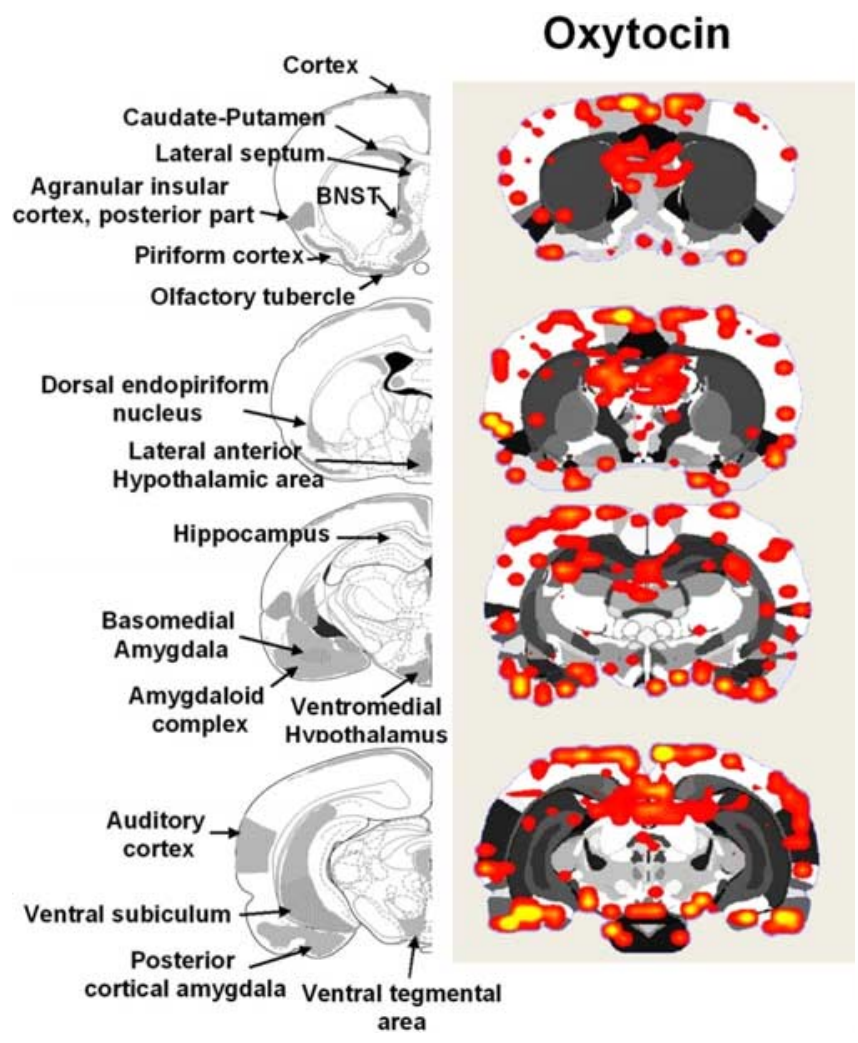

Figure 6. Brain areas showing positive BOLD responses to oxytocin in postpartum dams. Atlas coronal sections on the left highlight brain areas containing moderate-to-high levels of oxytocin receptors, as reported in the literature (moderate, gray colored areas; high, gray/ dotted areas) (Tribollet et al., 1988; Veinante and Freund-Mercier, 1997; Vaccari et al., 1998). The adjacent activation maps $(n=6)$ show regions of positive BOLD signal changes after administration of oxytocin. Colored pixels represent brain areas that showed signal intensity values significantly increased above baseline. Statistical significance was determined using a voxel-wise $t$ test analysis with false-positive detection filtering, comparing baseline and stimulation periods ( $\alpha=0.05$; Bonferroni corrected).

was not blocked by OTA nor induced by ICV OT) might be representative of an increase in the activity of inhibitory afferents to the VTA or an increase in the activity of inhibitory interneurons within the VTA. Therefore, although it is attractive to view the increased BOLD response in the VTA and NA as mediating reward-related aspects of suckling stimulation, it is also possible that changes in the activity of these systems are regulating motoric function during nursing so that the mother inhibits active voluntary responses and engages in responses that are more reflexive and inactive in nature.

In summary, the neuroanatomical substrates activated by oxytocin administration in the lactating rat closely paralleled that observed with suckling stimulation alone. Moreover, blockade of oxytocin receptors with a specific antagonist selectively reduced brain activity in many of these common areas, evidence that endogenous oxytocin has a role in the neurobiology of nursing. There were nonoverlapping brain regions, namely the caudateputamen, septum, and the thalamus, that could involve other neuropeptide and neurotransmitter systems independent of oxytocin release. These may include vasopressin, opioids, and dopamine. Understanding the neurobiology of maternal-infant bonding may improve the future clinical treatment of mothers afflicted by psychiatric disease states such as depression, anxiety, and drug addiction.

\section{References}

Byrnes EM, Rigero BA, Bridges RS (2002) Dopamine antagonists during parturition disrupt maternal care and the retention of maternal behavior in rats. Pharmacol Biochem Behav 73:869-875.

Champagne F, Diorio J, Sharma S, Meaney MJ (2001) Naturally occurring variations in maternal behavior in the rat are associated with differences in estrogen-inducible central oxytocin receptors. Proc Natl Acad Sci USA 98:12736-12741.

Febo M, Segarra AC, Tenney JR, Brevard ME, Duong TQ, Ferris CF (2004) Imaging cocaine-induced changes in the mesocorticolimbic dopaminergic system of conscious rats. J Neurosci Methods 139:167-176.

Ferris CF, Kulkarni P, Sullivan Jr JM, Harder JA, Messenger TL, Febo M (2005) Pup suckling is more rewarding than cocaine: evidence from functional magnetic resonance imaging and three-dimensional computational analysis. J Neurosci 25:149-156.

Fleming A, Vaccarino F, Tambosso L, Chee P (1979) Vomeronasal and olfactory system modulation of maternal behavior in the rat. Science 203:372-374.

Francis DD, Champagne FC, Meaney MJ (2000) Variations in maternal behaviour are associated with differences in oxytocin receptor levels in the rat. J Neuroendocrinol 12:1145-1148.

Genovese CR, Lazar NA, Nichols T (2002) Thresholding of statistical maps in functional neuroimaging using the false discovery rate. NeuroImage 15:870-878.

Hansen S, Bergvall AH, Nyiredi S (1993) Interaction with pups enhances dopamine release in the ventral striatum of maternal rats: a microdialysis study. Pharmacol Biochem Behav 45:673-676.

Insel TR, Harbaugh CR (1989) Lesions of the hypothalamic paraventricular nucleus disrupt the initiation of maternal behavior. Physiol Behav 45:1033-1041.

Kendrick KM, Da Costa AP, Broad KD, Ohkura S, Guevara R, Levy F, Keverne EB (1997) Neural control of maternal behaviour and olfactory recognition of offspring. Brain Res Bull 44:383-395.

King JA, Garelick TS, Brevard ME, Chen W, Messenger TL, Duong TQ, Ferris CF (2005) Procedure for minimizing stress for fMRI studies in conscious rats. J Neurosci Methods 148:154-160.

Lancel M, Krömer S, Neumann ID (2003) Intracerebral oxytocin modulates sleep-wake behaviour in male rats. Regul Pept 114:145-152.

Lee A, Li M, Watchus J, Fleming AS (1999) Neuroanatomical basis of maternal memory in postpartum rats: selective role for the nucleus accumbens. Behav Neurosci 113:523-538.

Levy F, Kendrick KM, Keverne EB, Piketty V, Poindron P (1992) Intracerebral oxytocin is important for the onset of maternal behavior in inexperienced ewes delivered under peridural anesthesia. Behav Neurosci 106:427-432.

Li C, Chen P, Smith MS (1999) Neural populations of the rat forebrain and brainstem activated by the suckling stimulus as demonstrated by cFos expression. Neuroscience 94:117-129.

Li M, Fleming AS (2003) The nucleus accumbens shell is critical for normal expression of pup-retrieval in postpartum female rats. Behav Brain Res 145:99-111.

Lin SH, Miyata S, Matsunaga W, Kawarabayashi T, Nakashima T, Kiyohara T (1998) Metabolic mapping of the brain in pregnant, parturient and lactating rats using Fos immunohistochemistry. Brain Res 787:226-236.

Lonstein JS, Stern JM (1997) Role of the midbrain periaqueductal gray in maternal nurturance and aggression: c-fos and electrolytic lesion studies in lactating rats. J Neurosci 17:3364-3378.

Lonstein JS, Simmons DA, Swann JM, Stern JM (1998) Forebrain expression of c-fos due to active maternal behaviour in lactating rats. Neuroscience 82:267-281.

Ludwig R, Bodgdanov G, King J, Allard A, Ferris CF (2004) A dual RF resonator system for high-field functional magnetic resonance imaging of small animals. J Neurosci Methods 132:125-135.

Mattson BJ, Williams S, Rosenblatt JS, Morrell JI (2001) Comparison of two positive reinforcing stimuli: pups and cocaine throughout the postpartum period. Behav Neurosci 115:683-694.

Neumann I, Russell JA, Landgraf R (1993a) Oxytocin and vasopressin release within the supraoptic and paraventricular nuclei of pregnant, parturient and lactating rats: a microdialysis study. Neuroscience 53:65-75.

Neumann I, Ludwig M, Engelmann M, Pittman QJ, Landgraf R (1993b) Simultaneous microdialysis in blood and brain: oxytocin and vasopressin 
release in response to central and peripheral osmotic stimulation and suckling in the rat. Neuroendocrinology 58:637-645.

Neumann I, Koehler E, Landgraf R, Summy-Long J (1994) An oxytocin receptor antagonist infused into the supraoptic nucleus attenuates intranuclear and peripheral release of oxytocin during suckling in conscious rats. Endocrinology 134:141-148.

Numan M (1994) A neural circuitry analysis of maternal behavior in the rat. Acta Paediatr Suppl 397:19-28.

Numan M (2004) Maternal behaviors: central integration or independent parallel circuits? Theoretical comments on Popeski and Woodside. Behav Neurosci 118:1469-1472.

Numan M, Corodimas KP (1985) The effects of paraventricular hypothalamic lesions on maternal behavior in rats. Physiol Behav 35:417-425.

Numan M, Numan MJ (1996) A lesion and neuroanatomical tract-tracing analysis of the role of the bed nucleus of the stria terminalis in retrieval behavior and other aspects of maternal responsiveness in rats. Dev Psychobiol 29:23-51.

Numan M, Smith HG (1984) Maternal behavior in rats: evidence for the involvement of preoptic projections to the ventral tegmental area. Behav Neurosci 98:712-727.

Numan M, Numan MJ, Schwarz JM, Neuner CM, Flood TF, Smith CD (2005) Medial preoptic area interactions with the nucleus accumbensventral pallidum circuit and maternal behavior in rats. Behav Brain Res 158:53-68.

Numan MJ, Insel TR (2003) The neurobiology of parental behavior, Ed 3. New York: Springer.

Paxinos G, Watson C (1997) The rat brain in stereotaxic coordinates, Vol 1, Ed 3. Boston: Academic.

Pedersen CA, Boccia ML (2003) Oxytocin antagonism alters rat dams' oral grooming and upright posturing over pups. Physiol Behav 80:233-241.

Pedersen CA, Prange Jr AJ (1979) Induction of maternal behavior in virgin rats after intracerebroventricular administration of oxytocin. Proc Natl Acad Sci USA 76:6661-6665.

Pedersen CA, Caldwell JD, Walker C, Ayers G, Mason GA (1994) Oxytocin activates the postpartum onset of rat maternal behavior in the ventral tegmental and medial preoptic areas. Behav Neurosci 108:1163-1171.

Pedersen CA, Johns JM, Musiol I, Perez-Delgado M, Ayers G, Faggin B, Caldwell JD (1995) Interfering with somatosensory stimulation from pups sensitizes experienced, postpartum rat mothers to oxytocin antagonist inhibition of maternal behavior. Behav Neurosci 109:980-990.

Price JL (1973) An autoradiographic study of complementary laminar patterns of termination of afferent fibers to the olfactory cortex. J Comp Neurol 150:87-108.

Simerly RB, Swanson LW (1988) Projections of the medial preoptic nucleus: a Phaseolus vulgaris leucoagglutinin anterograde tract-tracing in the rat. J Comp Neurol 270:209-242.

Slotnick BM, Nigrosh BJ (1975) Maternal behavior of mice with cingulate cortical, amygdala, or septal lesions. J Comp Physiol Psychol 88:118-127.

Stack EC, Balakrishnan R, Numan MJ, Numan M (2002) A functional neuroanatomical investigation of the role of the medial preoptic area in neural circuits regulating maternal behavior. Behav Brain Res 131:17-36.

Strupp JP (1996) Stimulate: a GUI based fMRI analysis software package. NeuroImage 3:S607.

Swanson LW (1999) Brain maps: structure of the rat brain, Ed 2. Boston: Elsevier Science.

Tribollet E, Barberis C, Dreifuss JJ, Jard S (1988) Autoradiographic localization of vasopressin and oxytocin binding sites in rat kidney. Kidney Int 33:959-965.

Vaccari C, Lolait SJ, Ostrowski NL (1998) Comparative distribution of vasopressin $\mathrm{V} 1 \mathrm{~b}$ and oxytocin receptor messenger ribonucleic acids in brain. Endocrinology 139:5015-5033.

van Leengoed E, Kerker E, Swanson HH (1987) Inhibition of post-partum maternal behaviour in the rat by injecting an oxytocin antagonist into the cerebral ventricles. J Endocrinol 112:275-282.

Veinante P, Freund-Mercier MJ (1997) Distribution of oxytocin- and vasopressin-binding sites in the rat extended amygdala: a histoautoradiographic study. J Comp Neurol 383:305-325.

Witt DM, Insel TR (1991) A selective oxytocin antagonist attenuates progesterone facilitation of female sexual behavior. Endocrinology 128:3269-3276.

Wu Z, Sullivan JMJ (2003) Multiple material marching cubes algorithm. IJNME 58:189-207.

Yoshimura R, Kiyama H, Kimura T, Araki T, Maeno H, Tanizawa O, Tohyama M (1993) Localization of oxytocin receptor messenger ribonucleic acid in the rat brain. Endocrinology 133:1239-1246. 